DOI: http://dx.doi.org/10.33846/hn31203

http://heanoti.com/index.php/hn

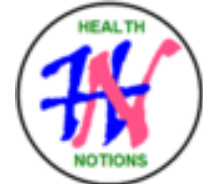

RESEARCH ARTICLE

URL of this article: http://heanoti.com/index.php/hn/article/view/hn31203

\title{
Pattern of Electroencephalography in Recurrent Febrile Seizure Patient
}

\author{
Adinda Chairunnisa $^{1(\mathrm{CA})}$, Prastiya Indra Gunawan ${ }^{2}$, Isti Suharjanti ${ }^{3}$ \\ ${ }^{1(\mathrm{CA})}$ Faculty of Medicine, Universitas Airlangga, Indonesia; adindachrnns@ gmail.com (Corresponding Author) \\ ${ }^{2}$ Department of Pediatrics, Faculty of Medicine, Universitas Airlangga, Indonesia; prastiya_ig@yahoo.co.id \\ ${ }^{3}$ Department of Neurology, Faculty of Medicine, Universitas Airlangga, Indonesia; isti.suharjanti@gmail.com
}

\begin{abstract}
Background: Febrile seizures are seizures that often occur in children, usually of a non-hazardous nature and do not have a prolonged effect. Febrile seizures most often occur in children under five years of age and are reported to occur in $2-5 \%$ of the pediatric population. Febrile seizures are categorized as simple, complex and plus febrile seizures. In some patients, EEG is needed to ascertain whether a true febrile seizure occurs. Objective: This study aims to determine the EEG pattern in recurrent febrile seizure patients at the Child Inpatient Installation of Dr. Soetomo Surabaya. Method: This study used a retrospective descriptive method with medical record instruments. Results: This study showed that of 46 recurrent febrile seizures, only 21 patients could see the EEG results. Of the 18 patients with complicated febrile seizures there were $27.78 \%$ abnormal and $72.22 \%$ normal. One simple febrile seizure patient obtained a normal EEG result. Of the two patients with febrile seizures plus 50\% abnormal results and 50\% normal results. Conclusions: The EEG pattern in patients with recurring complex febrile seizure obtains the most abnormal result.
\end{abstract}

Keywords: recurrent febrile seizure; electroencephalography; prevalence

\section{INTRODUCTION}

Febrile seizure most frequently occurs in children under five years old and is reported to occur in 2-5\% of the pediatric population. International League against Epilepsy (ILAE) defines febrile seizures as seizures that occurs in children or infants and are characterized by a body temperature above $38^{\circ} \mathrm{C}$ that is not caused by infection of the central nervous system (CNS). Children with febrile seizure usually lose consciousness, tremble, and move their whole body. The direct cause of febrile seizure remains unknown, but there are several important factors that potentially affect it; for instance fever, respiratory infections, hypoglycemia, hypocalcaemia, head trauma, poisoning and over-use of drugs, epilepsy, or gastroenteritis ${ }^{(1)}$.

There are 3 categories of febrile seizure, which are: simple, complex, and plus. Based on the latest evidences, a small proportion of children who have febrile seizure are more likely to have recurrent seizures or even epilepsy. According to a research in Korea, as many as $10 \%$ of children with febrile seizure are found to have additional febrile seizures and are later diagnosed with epilepsy ${ }^{(2)}$. There is no difference in criteria for children who can be affected by simple or complex febrile seizure, such as age, nerve conditions, or fever conditions. Children who have febrile seizure usually do not have significant neurological abnormalities both before and after the seizure. Febrile seizure can be categorized as simple if the seizures last briefly under 15 minutes and are comprehensive. Meanwhile, febrile seizure can be determined as complex if the seizures last for less than 15 minutes and are comprehensive. In other hand, febrile seizure is categorized as plus if the seizures last more than 15 minutes or occurs more than once during a fever in usually one point ${ }^{(3)}$.

Family history of febrile seizure, neonatal discharge for more than 28 days, developmental disorders, certain viral infections, and the possibility of iron and zinc deficiency are some of febrile seizure risk factors. Febrile seizures can occur before or shortly after the onset of fever ${ }^{(4)}$. It mostly occurs between the ages of 6 months to 5 years old with 18 months old as its peak.

Evaluation of febrile seizure can be done in several ways, such as laboratory tests, which are: Lumbar Puncture (LP) and complete blood tests, Electroencephalography (EEG), and Magnetic Resonance Imaging (MRI). However, not all febrile seizures require evaluation, such as simple febrile seizure. The evaluation is 
only carried out if the child has a complex febrile seizure or there is a suspicion that the febrile seizure can develop into epilepsy ${ }^{(3)}$.

\section{METHODS}

This research was a retrospective descriptive study by collecting medical records or from secondary data from patients. The technique of taking research subjects using a total sampling technique. The study comprised 46 children at the RSUD Dr. Soetomo who presented with febrile seizures. We analyzed data from the inpatient installation of Dr. Soetomo for the period from January 1, 2017 to 31 June, 2018 with exclusion criteria for incomplete medical record data. The data obtained were analyzed using the observational analysis method and presented descriptively.

\section{RESULTS}

Based on the results of the study, out of 137 medical record data obtained, only 46 patients experienced recurrent febrile seizure and only 21 patients obtained the results of the EEG examination.

\section{Age and Gender Distribution of Patients with Recurrent Febrile Seizure}

Table 1 shows that from a total of 46 patients with recurrent febrile seizure at RSUD Dr. Soetomo, the youngest age was 8 months and the oldest was 9 years old. The age distribution of most recurrent febrile seizure is in the age range of 1-3 years as many as 29 children (63.04\%). Table 1 also shows that recurrent febrile seizure were more common in boys, with a percentage of $63.04 \%$, out of the total of 46 patients with recurrent febrile seizure at RSUD Dr. Soetomo.

Table 1. Age and gender distribution of patients with recurrent febrile seizure

\begin{tabular}{cc}
\hline Variables & $\mathrm{N}(\%)$ \\
\hline Age (year) & \\
\hline$<1$ & $7(15.22)$ \\
$1-3$ & $29(63.04)$ \\
$3-5$ & $5(10.87)$ \\
$>5$ & $5(10.87)$ \\
\hline Total & $46(100)$ \\
\hline Gender & \\
\hline Girls & $29(63.04)$ \\
Boys & $17(36.96)$ \\
\hline Total & $46(100)$ \\
\hline
\end{tabular}

\section{Distribution of Types of Recurrent Febrile Seizure}

Febrile seizure divided in three categories which are complex, simple, and plus febrile seizure. The diagram above shows that from a total of 46 patients with recurrent febrile seizure, complex febrile seizure is the most common type (72\%) in RSUD Dr. Soetomo.

Table 2. Distribution of types of recurrent febrile seizure

\begin{tabular}{cc}
\hline Type of febrile seizure & N (\%) \\
\hline Simple FS & $11(24)$ \\
Complex FS & $33(72)$ \\
FS Plus & $2(4)$ \\
\hline Total & $46(100)$ \\
\hline
\end{tabular}

\section{Distribution of EEG Examination Result}

From a total of 46 patients with recurrent febrile seizure at RSUD Dr. Soetomo, only 21 patients had their EEG examination results and only 6 patients resulting abnormal in EEG examination. The abnormal results are most common in complex febrile seizure that followed by plus febrile seizure. Meanwhile, in simple febrile seizure no abnormal results are obtained. 
Table 3. Distribution of EEG examination result

\begin{tabular}{lc}
\hline Febrile serizure types & $\mathrm{N}(\%)$ \\
\hline Complex FS & \\
\hline Normal & $13(72,22)$ \\
Abnormal I & $2(11,11)$ \\
Abnormal II & $2(11,11)$ \\
Abnormal III & $1(5,56)$ \\
\hline Total & $18(100)$ \\
\hline Simple FS & $1(100)$ \\
\hline Normal & $0(0)$ \\
Abnormal I & $0(0)$ \\
Abnormal II & $0(0)$ \\
Abnormal III & $1(100)$ \\
\hline Total & \\
\hline FS Plus & $1(50)$ \\
\hline Normal & $1(50)$ \\
Abnormal I & $0(0)$ \\
Abnormal II & $0(0)$ \\
Abnormal III & $2(100)$ \\
\hline Total &
\end{tabular}

From a total of 46 medical records of recurrent febrile seizure patients at RSUD Dr. Soetomo, the youngest age was 8 months and the oldest was 9 years. According to the age distribution of recurrent febrile seizure patients, it is mostly found in the group of 1-3 years old, as many as 29 children (63.04\%), followed by the age group under 1 year, as many as 7 children (15.22\%). While those in the age range of 3-5 years old and group over 5 years old occupying the same position, with each of them as many as 5 children (10.87\%).

Research conducted in India divided children into two groups, which are 1 month to 3 years and 3-6 years. From a total of 545 children, there are 333 children $(61 \%)$ aged 1 month to 3 years and 212 children (39\%) aged 3-6 years, which means children with febrile seizure are more common in children under the age of 3 years ${ }^{(5)}$.

Another study conducted in Korea divided children into five groups, which are: less than 1 year, 1 year, 2 years, 3 years, and 4 years. Mostly, febrile seizures are found in the 2-year age group, which is $27.5 \%$. However, for recurrent febrile seizure, there are more common to be found in the 3-year age group ${ }^{(6)}$. This indicates that there is a majority of age differences between first febrile seizure and recurrent febrile seizure.

According to research conducted in America, out of a total of 347 children with febrile seizure, 94 of children $(27 \%)$ had recurrent febrile seizure. The risk of recurrence is $14 \%$ in children aged 24 months ${ }^{(7)}$. The results of several studies that have been carried out in various countries have similar results, which the peak is in the age range of 1-3 years.

Fever seizures are divided into three categories, namely: complicated febrile seizure, simple febrile seizure, and plus febrile seizure. The diagram above shows that from a total of 46 patients with recurrent febrile seizure at RSUD Dr. Soetomo, the most types of febrile seizure were complicated febrile seizure, as many as 33 patients $(72 \%)$, followed by simple febrile seizure in 11 patients $(24 \%)$, and plus febrile seizure, as many as 2 patients $(4 \%)$.

Research results in several journals have different results. In a study conducted in Indonesia, precisely at RSHS Bandung, out of 58 children with recurrent febrile seizure, $38(65 \%)$ of those suffer complicated febrile seizure, and 16 children (28\%) sufer simple febrile seizure, while in 4 children (7\%) the type of febrile seizure cannot be identified $^{(8)}$. This is in similar to the results of this study.

The results of another study conducted in India, out of 288 patients were found as many as 231 children $(80.2 \%)$ with a diagnosis of simple febrile seizure, and $57(19.8 \%)$ children with a diagnosis of complicated febrile seizure ${ }^{(9)}$. In another study conducted in Iran, patients with complicated febrile seizure were as many as 63 children $(18.86 \%)$ and simple febrile seizure were $271(81.14 \%)$ out of 334 children in total $^{(10)}$. This study is only focusing on patients with recurrent febrile seizure that might cause the different results. Meanwhile, the other studies included all febrile seizures, both first and recurrent febrile seizures.

Out of 46 patients with recurrent febrile seizure at RSUD Dr. Dr. Soetomo, only 21 patients obtained the results of the EEG examination. From the 21 patients only 6 patients were found with abnormal EEG examination results. After seeing the distribution of abnormal and normal results from the three groups of patients with simple febrile seizure, complex febrile seizure and plus febrile seizure; Abnormal results are most 
common in patients with complicated febrile seizure, as many as 5 patients and followed by plus febrile seizure as many as 1 patient, while in simple febrile seizure no abnormal results are obtained.

The abnormal results obtained in complicated fever seizure, which as much as $27.78 \%$, is divided into abnormal I (2 patients), abnormal II (2 patients), and abnormal III. A normal result as much as $72.22 \%$ shows that normal results predominate in complicated febrile seizure. In two of the EEG abnormal complicated febrile seizure were found Excessive Beta impression results indicating mild diffuse encephalopathy. In addition, one of the EEG abnormal results in complicated febrile seizure obtained the Background Slow Activity impression that indicates the presence of moderate diffuse encephalopathy that potentially generates general epileptogenic.

This research is similar to some of the literature that has been done. A study in Romania stated that abnormal EEG results were obtained in all patients with a type of complicated febrile seizure (100\%) in as many as 12 patients, compared with simple febrile seizure which only found as many as $30(31 \%)$ abnormal results out of 96 patients with simple febrile seizure ${ }^{(11)}$.

The results of this study indicate recurrent febrile seizures at Dr. Soetomo Surabaya are more common in boys. Out of 46 children with recurrent febrile seizure, were found that as many as $29(63.04 \%)$ are boys and 17 $(36.96 \%)$ are girls.

Some journals have the same majority result, which boys dominate febrile seizure. Research conducted in Bandung states that boys have a greater percentage of recurrent febrile seizure than girls, which is $72 \%$ boys and $28 \%$ girls $^{(7)}$ indriai . Another study conducted in India results $308(56.5 \%)$ boys and $237(43.5 \%)$ girls with a diagnosis of febrile seizure, both recurrent and not ${ }^{(5)}$.

\section{CONCLUSION}

EEG examination results in patients with recurrent febrile seizures obtained the most abnormalities in complicated febrile seizures followed by febrile seizures plus, while in simple febrile seizures there are no abnormalities. The results of this study generally have similarities with studies that have been carried out before all over the world.

For the development of science, it is better if in the future it will conduct research with the same purpose, using primary and secondary data as well as research material, so that more information can be obtained and minimize blank data. The use of descriptive research methods around febrile seizures also needs to be increased because there is still little information available, especially in Indonesia.

\section{REFERENCES}

1. Delpisheh A, Veisani Y, Sayehmiri K, Fayyazi A. Febrile Seizures: Etiology, Prevalence, and Geographical Variation. Iran J Child Neurol. 2014;8(3):30-37.

2. Lee S, Byeon J, Kim G, Eun B, and Eun S. Epilepsy in children with a history of febrile seizures. Korean Journal of Pediatrics. 2016;59(2):74-79.

3. Seinfeld D, Pellock J. Recent Research on Febrile Seizures: A Review. J Neurol Neurophysiol. 2014;4(165):1-14.

4. Graves R, Oehler K, Tingle L. Febrile Seizures: Risks, Evaluation, and Prognosis. American Family Physician. 2012;85(2):149-153.

5. Naik S, Jan M, Rafiq W, Ahmad S, Maqbool M. Febrile seizures in preschool children Kashmir India. International Journal of Contemporary Pediatrics. 2015;2(3):213-215.

6. Byeon J, Kim G, Eun B. Prevalence, Incidence, and Recurrence of Febrile Seizures in Korean Children Based on National Registry Data. Journal of Clinical Neurology. 2018;14(1):43.

7. Berg A, Shinnar S, Hauser W, Alemany M, Shapiro E, Salomon M, et al. A Prospective Study of Recurrent Febrile Seizures [Internet]. New England Journal of Medicine. 1992;327(16), [cited 2019 June 13]. p. 11221127. Available from: https://www.nejm.org/doi/full/10.1056/NEJM199210153271603

8. Indriani A, Risan N, Nurhayati T. Five Years Study of Recurrent Febrile Seizure Risk Factors. Althea Medical Journal. 2017;4(2):282-285.

9. Potdar P. A retrospective study of febrile seizures among children admitted in a tertiary care hospital. International Journal of Community Medicine and Public Health. 2018;5(7):3121-3124.

10. Eskandarifar A, Fatolahpor A, Asadi G, Gaderi I. The Risk Factors in Children with Simple and Complex Febrile Seizures: An Epidemiological Study. Int J Pediatr. 2017;5(6):5137-44.

11. Costea R, Neamţu B, Maniu G. Pathological EEG Pattern, Predictive Factor for Recurrence or Epilepsy in Febrile Seizures?. Acta Medica Transilvanica. 2016;21(4):22-26. 